\title{
Risk factors for acute kidney injury in neonates with congenital diaphragmatic hernia
}

\author{
Brianna M. Liberio $\mathbb{1}^{1} \cdot$ John T. Brinton ${ }^{2} \cdot$ Katja M. Gist $\mathbb{B}^{3} \cdot$ Danielle E. Soranno ${ }^{4} \cdot$ Megan J. Kirkley $\mathbb{1}^{1,5}$. \\ Jason Gien (10)
}

Received: 11 January 2021 / Revised: 13 May 2021 / Accepted: 25 May 2021 / Published online: 12 June 2021

(c) The Author(s), under exclusive licence to Springer Nature America, Inc. 2021

\begin{abstract}
Objective To examine incidence of acute kidney injury (AKI), antenatal and postnatal predictors, and impact of AKI on outcomes in infants with congenital diaphragmatic hernia (CDH).

Study design Single center retrospective study of 90 CDH infants from 2009-2017. Baseline characteristics, CDH severity, possible AKI predictors, and clinical outcomes were compared between infants with and without AKI.

Result In total, 38\% of infants developed AKI, 44\% stage 1, 29\% stage 2, 27\% stage 3. Lower antenatal lung volumes and liver herniation were associated with AKI. Extracorporeal life support (ECLS), diuretics, abdominal closure surgery, hypotension, and elevated plasma free hemoglobin were associated with AKI. Overall survival was 79\%, 47\% with AKI, and 35\% with AKI on ECLS. AKI is associated with increased mechanical ventilation duration and length of stay.

Conclusion AKI is common among $\mathrm{CDH}$ infants and associated with adverse outcomes. Standardized care bundles addressing AKI risk factors may reduce AKI incidence and severity.
\end{abstract}

\section{Introduction}

Congenital diaphragmatic hernia $(\mathrm{CDH})$ is a severe birth anomaly occurring in 1:3000 live births, where a defect in the diaphragm allows abdominal organs to herniate into the thorax, compressing the intrathoracic structures [1]. The defect forms between 8-10 weeks of gestation, compromising growth and development of the lungs and heart

Brianna M. Liberio

Brianna.liberio@childrenscolorado.org

1 Department of Pediatrics, Section of Neonatology, Children's Hospital Colorado, University of Colorado Anschutz Medical Campus, Aurora, CO, USA

2 Department of Biostatistics and Informatics, Colorado School of Public Health, Aurora, CO, USA

3 Department of Pediatrics, Section of Cardiology, Children's Hospital Colorado, University of Colorado Anschutz Medical Campus, Aurora, CO, USA

4 Department of Pediatrics, Section of Nephrology, Children's Hospital Colorado, University of Colorado Anschutz Medical Campus, Aurora, CO, USA

5 Department of Pediatrics, Denver Health Medical Center, Denver, CO, USA in utero, resulting in lung parenchyma and vascular abnormalities [2]. About one-third of infants with $\mathrm{CDH}$ require extracorporeal life support (ECLS), representing the most severe lesions [3]. While the overall survival rate for isolated CDH ranges from 50-80\%, infants requiring ECLS have worse survival rates, with almost six times increased odds of mortality [2-5].

Risk factors for adverse outcomes in neonates with $\mathrm{CDH}$ include low birth weight, liver herniation into the chest, lung hypoplasia, pulmonary hypertension, left ventricular hypoplasia, chromosomal anomalies, and congenital heart disease [3]. With the advent of fetal magnetic resonance imaging (MRI) and high-resolution obstetrical ultrasound, accuracy of antenatal assessments of severity of lung hypoplasia has markedly improved. Ultrasound assessment of lung-head ratio (LHR) and observed to expected lung-head ratio (O:E LHR), as well as MRI assessment of percent predicted lung volume (PPLV) and fetal total lung volume (TLV) are the gold standards for predicting the degree of lung hypoplasia [6-9]. Significant hypoplasia, suggested by lower LHR, O:E LHR, PPLV, and TLV values, is associated with an incremental increase in ECLS utilization and a decrease in survival rates [8-11].

Acute kidney injury (AKI) is common in critically ill adults and children, and it is an independent risk factor for 
mortality in these populations $[12,13]$. The Assessment of Worldwide Acute Kidney Epidemiology in Neonates (AWAKEN) Study (a large, multi-center observational cohort study) reported an AKI incidence of 30\% across all gestational ages in the neonatal intensive care unit population and an independent association between AKI and mortality [14]. AKI has been studied in specific neonatal populations. Ryan et al. reported an AKI incidence of $37 \%$ in a cohort of 54 infants with CDH [15], and Gadepalli et al. reported an incidence of $71 \%$ in those with severe $\mathrm{CDH}$ requiring ECLS [4]. Renal failure was associated with decreased ventilator-free days in the first 60 days of life and increased ECLS duration [4]. Due to the complexity of $\mathrm{CDH}$, this population represents a unique group of neonates with increased risk for both AKI and mortality.

While there is evidence linking AKI to poor outcomes in $\mathrm{CDH}$, the risk factors responsible for the development of AKI in infants with CDH remain unclear. In this study of infants with $\mathrm{CDH}$, we aimed to (1) determine the incidence of AKI, (2) examine antenatal characteristics and postnatal exposures associated with AKI, and (3) determine the impact of AKI on long-term outcomes, including length of stay, duration of mechanical ventilation, and mortality. We hypothesized that, in agreement with existing literature, AKI would be common in the $\mathrm{CDH}$ population, with an increased incidence in those requiring ECLS. In addition, we hypothesized that antenatal measures suggesting worse $\mathrm{CDH}$ severity and the presence of significant postnatal exposures, including nephrotoxins, sepsis, surgery, ECLS, and elevated plasma free hemoglobin, would confer an increased risk for AKI.

\section{Materials and methods}

Single center retrospective study of inborn and outborn infants with $\mathrm{CDH}$, managed at the Level IV Neonatal Intensive Care Unit at Children's Hospital Colorado, from April 2009 to November 2017. Patients were identified and data extracted through review of the electronic health record. This study was approved by the Colorado Multiple Institutional Review Board with a waiver of informed consent.

Extracted data included demographics; antenatal measures of $\mathrm{CDH}$ severity, including presence or absence of liver herniation, PPLV, TLV, LHR, and O:E LHR as available from prenatal imaging for inborn patients; presence of congenital heart disease, congenital anomalies of the kidney and urinary tract, and chromosomal anomalies. Congenital heart disease was defined as structurally abnormal cardiac anatomy, excluding patent ductus arteriosus, patent foramen ovale, atrial septal defect, and small muscular ventricular septal defect.
For those requiring ECLS, the day of initiation and duration were documented. All ECLS was veno-arterial support using the Maquet centrifugal pump, with the same system used over the duration of the study period. Use of continuous renal replacement therapy (CRRT) was defined as a dichotomous outcome. The most common indication for CRRT use at our institution is metabolic control of uremia, with blood urea nitrogen $>150 \mathrm{mg} / \mathrm{dL}$. Use of nephrotoxins, including vancomycin, gentamicin, loversol, and acyclovir, and the use of diuretics, including furosemide, chlorothiazide, and bumetanide, were extracted. Antibiotics and antivirals were administered based on dosing recommendations in Lexicomp, and antibiotic peak and trough levels were monitored per recommendations. Diuretics were intermittently dosed. Median days of exposure were calculated. Presence of positive blood cultures was extracted.

Timing of $\mathrm{CDH}$ and abdominal closure surgery was extracted. At our institution, for all patients requiring ECLS, CDH repair occurs in first 24-48 hours after ECLS cannulation. Indications for delay of repair include coagulopathy, moderate or severe left ventricular dysfunction, and hemodynamic instability defined as need for epinephrine $>0.1 \mathrm{mcg} / \mathrm{kg} / \mathrm{min}$ or vasopressin $>0.001 \mathrm{units} / \mathrm{kg} / \mathrm{min}$. The decision for abdominal closure is at the discretion of the surgeon and not based on a pre-defined protocol. Mean arterial pressure (MAP), central venous pressure, and renal perfusion pressure (MAP-central venous pressure) were assessed 3 days prior to and 3 days after $\mathrm{CDH}$ and abdominal closure surgery. A "normal MAP" was defined by gestational age. In all patients with $\mathrm{CDH}$, large bore (20 gauge) central venous catheters are placed by cut-down method, allowing for accurate measurement of central venous pressure. Finally, multiple plasma free hemoglobin levels are measured daily for patients on ECLS at our institution, however we extracted the highest daily value for analysis in this study based on prior literature describing associations between elevated plasma free hemoglobin and AKI [16-18].

Fluid intake and output data were collected, and daily net fluid balance was utilized to perform a fluid-corrected serum creatinine ( $\mathrm{SCr}$ ) calculation, as detailed below. Date of birth was designated as day of life 0 , and data was collected daily through day of life 30 . Daily $\mathrm{SCr}$ values, when available, were extracted for the first 30 days of life. If more than one $\mathrm{SCr}$ value was reported in one day, the highest value was used. AKI was defined and staged using the $\mathrm{SCr}$ criteria of the Neonatal Modified Kidney Disease: Improving Global Outcomes (KDIGO) AKI definition $[19,20]$ (Table 1). The urine output (UOP) criteria of the Neonatal Modified KDIGO AKI definition was not used. Some patients had multiple episodes of AKI within the first 30 days of life. An episode of AKI was defined as 
Table 1 Neonatal Modified Kidney Disease: Improving Global Outcomes (KDIGO) AKI definition (Reference $\mathrm{SCr}$ is the lowest prior $\mathrm{SCr}$ measurement $[19,20])$.

\begin{tabular}{lll}
\hline Stage & Serum Creatinine & Urine output over $24 \mathrm{~h}$ \\
\hline 0 & No change in $\mathrm{SCr}$ or rise $<0.3 \mathrm{mg} / \mathrm{dL}$ & $>1 \mathrm{~mL} / \mathrm{kg} / \mathrm{hr}$ \\
1 & $\begin{array}{l}\text { SCr rise } \geq 0.3 \mathrm{mg} / \mathrm{dL} \text { within } 48 \mathrm{~h} \text { or SCr rise } \geq 1.5-1.9 \text { X reference } \mathrm{SCr} \\
\text { within } 7 \text { days }\end{array}$ & $>0.5$ and $\leq 1 \mathrm{~mL} / \mathrm{kg} / \mathrm{hr}$ \\
2 & SCr rise $\geq 2-2.9$ X reference $\mathrm{SCr}$ & $>0.3 \mathrm{and} \leq 0.5 \mathrm{~mL} / \mathrm{kg} / \mathrm{hr}$ \\
3 & SCr rise $\geq 3$ X reference $\mathrm{SCr}$ or $\mathrm{SCr} \geq 2.5 \mathrm{mg} / \mathrm{dL}$ or receipt of dialysis & $\leq 0.3 \mathrm{~mL} / \mathrm{kg} / \mathrm{hr}$ \\
\hline
\end{tabular}

$\mathrm{SCr}$ serum creatinine. recovered when the fluid-corrected $\mathrm{SCr}$ reached $110 \%$ of the prior baseline fluid-corrected $\mathrm{SCr}$ [19]. SCr, and thus $\mathrm{AKI}$, was corrected for fluid balance based on the equation below [21].

Fluid Corrected SCr $=$ Measured SCr $\times[1+$ (accumulated net fluid balance $\div$ total bodywater $)]$

Accumulated net fluid balance in liters, extracted from daily 24-h fluid intake and output data

Total body water defined as: $0.8 \times$ birth weight $(\mathrm{kg})$.

\section{Statistical methods}

Summary statistics included frequency (percent), mean (standard deviation), or median (interquartile range (IQR), when descriptive plots indicated skewed data). Wilson confidence intervals were calculated for estimated proportions of interest. Baseline characteristics were compared between those with and without AKI via $t$-tests, Wilcoxon Rank Sum test, or Chi-Squared as appropriate. Multiple nonlinear regression models assessed the associations between AKI during the first 30 days of life and predictors of interest (exposure to ECLS, nephrotoxins, diuretics; CDH repair surgery; abdominal closure surgery; positive blood culture; MAP; plasma-free hemoglobin value). For individuals who experienced multiple episodes of AKI, each episode was analyzed individually. Both univariable regression models and models adjusting for ECLS assessed the association between the binary longitudinal outcome of AKI status and each predictor of interest via generalized estimating equations. Knowing that changes in $\mathrm{SCr}$ can be delayed between the exposure and time to AKI diagnosis [22], we utilized a lagged predictor model to evaluate the impact of certain predictors prior to a diagnosis of AKI. For each predictor, 24-, 48-, and 72-hour lags were applied. All models assumed an auto-regressive correlation structure with a logit link function. Estimated associations between predictors and AKI are presented as odds ratios (OR) with $95 \%$ confidence intervals (CI). Survival was compared via Fisher's Exact or Chi-Squared tests and presented as relative risk (RR) with 95\% CI. Among survivors, days on mechanical ventilation and length of stay were compared via Jonckheere-Terpstra trend test to account for ordering of the outcomes. Statistical significance was set at an alpha 0.05 level. No adjustment for multiple comparisons were made. The data analysis was generated using SAS software version 9.4. Copyright (C) 2002-2012 by SAS Institute Inc. Cary, NC, USA.

\section{Results}

\section{Incidence of AKI}

Ninety infants with CDH were included. Median gestational age was 38 weeks [IQR: 36, 38], median birthweight was $2.89 \mathrm{~kg}$ [IQR: $2.5,3.19], 56 \%$ were male, and $50 \%$ were outborn. AKI occurred in $34(37.8 \%)$ infants during the first 30 days of life, $44 \%$ with stage 1 AKI, $29 \%$ stage 2 AKI, and $27 \%$ stage 3 AKI (Fig. 1a). Thirty-two infants (36\%) required ECLS, and AKI occurred in $26(81 \%)$ of these infants. The median day of AKI diagnosis was day of life 12 [IQR: 7.5, 19.5] (Fig. 1b). Nine infants had two episodes of AKI, and two infants had three episodes of AKI. When including only the first episode of AKI, the median day of AKI diagnosis was day of life 10 [IQR: 6, 14]. Eight patients required CRRT for metabolic control of uremia and fluid removal for anuria or oliguria, most commonly. Half of these patients had stage 3 AKI per the $\mathrm{SCr}$ criteria of the Neonatal Modified KDIGO AKI definition (Table 1) prior to requiring CRRT.

\section{Antenatal and postnatal exposures associated with AKI}

Infants who developed AKI had lower antenatal assessments of fetal lung volume (PPLV, TLV, LHR, and O:E LHR) and higher occurrences of liver herniation into the chest when compared to infants without AKI. Of those infants with AKI, 85\% had liver herniation, mean PPLV 17.5\%, median TLV $25 \mathrm{~mL}$, median LHR 1 , and median O: E LHR $38.5 \%$ (Table 2). Gestational age, birthweight, gender, inborn versus outborn status, and presence of congenital heart disease, chromosomal anomaly, or congenital anomalies of the kidney and urinary tract were not significantly different between those with and without AKI (Table 2). From an unadjusted analysis, those requiring 
a.

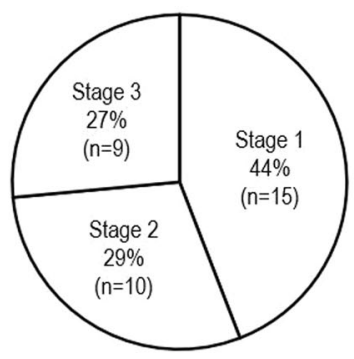

b.

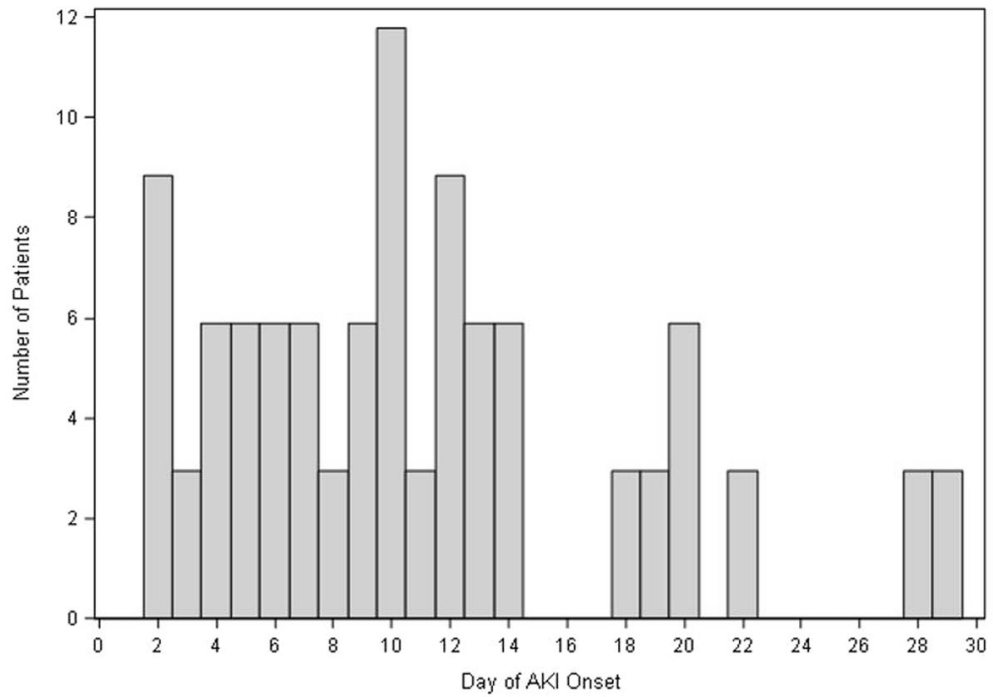

Fig. 1 Incidence and Timing of AKI. a Stratification by stage of AKI. Total number of patients with AKI is 34, representing $37.8 \%$ of the entire cohort. Upon stratification by stage of AKI, there was $44 \%$ with stage 1 AKI, $29 \%$ stage 2 AKI, and $27 \%$ stage 3 AKI. b Distribution of day of AKI onset. The median day of AKI diagnosis was day of life 12 [IQR: 7.5, 19.5]. AKI acute kidney injury.
Table 2 Associations between antenatal characteristics and patient demographics with occurrence of AKI.

\begin{tabular}{|c|c|c|c|}
\hline Prenatal characteristics & No AKI $(n=56)$ & AKI $(n=34)$ & $p$ value \\
\hline Left-sided CDH, $n(\%)$ & $47(83.9)$ & $27(79.4)$ & 0.80 \\
\hline Percent predicted lung volume, mean (SD) & $25.71(8.00)$ & $17.45(7.34)$ & $<0.001$ \\
\hline Total lung volume (mL), median [IQR] & $31.00[26.75,41.00]$ & $25.00[21.00,32.50]$ & $<0.05$ \\
\hline Lung-to-head ratio, median [IQR] & $1.40[1.10,1.60]$ & $1.00[0.80,1.10]$ & $<0.001$ \\
\hline $\begin{array}{l}\text { Observed to expected lung-to-head ratio, } \\
\text { median [IQR] }\end{array}$ & $52.00[39.75,66.00]$ & $38.50[24.90,48.00]$ & $<0.01$ \\
\hline Liver up, $n(\%)$ & $29(51.8)$ & $29(85.3)$ & $<0.01$ \\
\hline Patient demographics & No AKI $(n=56)$ & AKI $(n=34)$ & $p$ value \\
\hline GA (week), median [IQR] & $38.00[36.00,38.00]$ & $38.00[36.00,39.00]$ & 0.41 \\
\hline Birth weight $(\mathrm{kg})$, median [IQR] & $2.95[2.50,3.28]$ & $2.79[2.50,3.04]$ & 0.38 \\
\hline Male gender, $n(\%)$ & $31(55.4)$ & $19(55.9)$ & $>0.99$ \\
\hline Congenital heart disease, $n(\%)$ & $2(3.6)$ & $5(14.7)$ & 0.13 \\
\hline Chromosomal anomaly, $n(\%)$ & $2(3.6)$ & $3(8.8)$ & 0.56 \\
\hline $\begin{array}{l}\text { Congenital anomaly of the kidney or urinary tract, } \\
n(\%)\end{array}$ & $3(5.4)$ & $2(5.9)$ & $>0.99$ \\
\hline Outborn, $n(\%)$ & $31(55.4)$ & $14(41.2)$ & 0.28 \\
\hline ECLS use, $n(\%)$ & $6(10.7)$ & $26(76.5)$ & $<0.001$ \\
\hline
\end{tabular}

$A K I$ acute kidney injury, $C D H$ congenital diaphragmatic hernia, $G A$ gestational age, $E C L S$ extracorporeal life support.

ECLS had a 5.1 increased risk for AKI (95\% CI: 3.8-6.8) compared to those who did not require ECLS.

Among the eight patients who received CRRT, 75\% were inborn and had antenatal CDH severity measurements available for analysis. Mean PPLV 12.5\%, median TLV $22 \mathrm{~mL}$, median LHR 0.88, median O:E LHR 33.4\%, and all patients had liver herniation into the thorax. All patients received ECLS, and $88 \%$ received at least one diuretic prior to CRRT initiation.
Among the cohort, the median exposure days for nephrotoxins, including vancomycin, gentamicin, and acyclovir, was 2 days [IQR: 2, 5]. The median exposure days of diuretics, including furosemide, chlorothiazide, and bumetanide, was 5 days [IQR: 2, 14]. Using the lagged predictors model for univariable associations between postnatal exposures and the development of AKI, use of ECLS at 72 and $24 \mathrm{~h}$ prior to and on the day of AKI was associated with 
Table 3 Associations between postnatal exposures with occurrence of AKI, (a) unadjusted ${ }^{\mathrm{a}}$ and (b) adjusted for ECLS use ${ }^{\mathrm{b}}$.

\begin{tabular}{|c|c|c|c|c|c|c|c|c|}
\hline \multirow[b]{2}{*}{ Postnatal exposure } & \multicolumn{8}{|c|}{ Odds ratio $(95 \% \mathrm{CI})$} \\
\hline & Day of AKI onset & $p$ value & $24 \mathrm{~h}$ prior to $\mathrm{AKI}$ & $p$ value & $48 \mathrm{~h}$ prior to $\mathrm{AKI}$ & $p$ value & $72 \mathrm{~h}$ prior to $\mathrm{AKI}$ & $p$ value \\
\hline \multicolumn{9}{|l|}{ (a) Unadjusted } \\
\hline ECLS & $1.69(1.02-2.81)$ & $<0.05$ & $1.73(1.14-2.63)$ & $<0.01$ & $0.99(0.62-1.58)$ & 0.96 & $2.57(1.56-4.22)$ & $<0.01$ \\
\hline Nephrotoxin exposure (any) & $1.09(0.82-1.43)$ & 0.56 & $1.15(0.85-1.55)$ & 0.36 & $1.11(0.86-1.44)$ & 0.44 & $0.89(0.70-1.14)$ & 0.37 \\
\hline Vancomycin & $1.16(0.85-1.59)$ & 0.35 & $1.20(0.85-1.69)$ & 0.29 & $1.19(0.87-1.62)$ & 0.28 & $0.85(0.66-1.11)$ & 0.24 \\
\hline Gentamicin & $0.79(0.62-1.01)$ & 0.07 & $0.81(0.64-1.01)$ & 0.06 & $0.83(0.67-1.04)$ & 0.11 & $1.09(0.77-1.54)$ & 0.64 \\
\hline Diuretic exposure (any) & $1.56(1.03-2.36)$ & $<0.05$ & $1.65(1.07-2.53)$ & $<0.05$ & $1.30(0.82-2.07)$ & 0.26 & $1.10(0.72-1.67)$ & 0.67 \\
\hline Furosemide & $1.51(1.01-2.26)$ & $<0.05$ & $1.59(1.03-2.46)$ & $<0.05$ & $1.35(0.88-2.08)$ & 0.16 & $1.08(0.70-1.65)$ & 0.74 \\
\hline Chlorothiazide & $2.05(0.63-6.61)$ & 0.23 & $3.26(1.30-8.19)$ & $<0.05$ & $2.67(1.06-6.75)$ & $<0.05$ & $2.46(1.03-5.88)$ & $<0.05$ \\
\hline $\begin{array}{l}\mathrm{CDH} \text { repair surgery in } \\
\text { general }\end{array}$ & $1.19(0.92-1.55)$ & 0.19 & $1.12(0.76-1.65)$ & 0.56 & $0.83(0.56-1.21)$ & 0.32 & $1.14(0.92-1.41)$ & 0.24 \\
\hline $\begin{array}{l}\text { CDH repair surgery } \\
\text { on ECLS }\end{array}$ & $1.33(0.76-2.35)$ & 0.32 & $1.21(0.71-2.06)$ & 0.48 & $1.02(0.68-1.52)$ & 0.94 & $0.94(0.64-1.37)$ & 0.74 \\
\hline Abdominal closure surgery & $1.35(1.08-1.70)$ & $<0.01$ & $1.00(0.70-1.42)$ & 0.99 & $0.94(0.68-1.29)$ & 0.69 & $1.06(0.87-1.30)$ & 0.54 \\
\hline Positive blood culture & $2.05(0.81-5.18)$ & 0.13 & $1.04(0.95-1.13)$ & 0.37 & $1.03(0.96-1.11)$ & 0.45 & $0.42(0.04-4.37)$ & 0.46 \\
\hline $\begin{array}{l}\text { Mean arterial pressure } \\
\text { decrease }\end{array}$ & $1.03(1.01-1.06)$ & 0.01 & $1.00(0.97-1.02)$ & 0.75 & $1.01(0.98-1.04)$ & 0.70 & $1.00(0.97-1.04)$ & 0.91 \\
\hline \multicolumn{9}{|l|}{ (b) Adjusted for ECLS use } \\
\hline Nephrotoxin exposure (any) & $1.11(0.86-1.43)$ & 0.42 & $1.14(0.86-1.51)$ & 0.35 & $1.10(0.86-1.41)$ & 0.46 & $0.90(0.71-1.15)$ & 0.39 \\
\hline Vancomycin & $1.21(0.91-1.61)$ & 0.20 & $1.22(0.88-1.68)$ & 0.23 & $1.21(0.91-1.63)$ & 0.19 & $0.88(0.69-1.12)$ & 0.29 \\
\hline Gentamicin & $0.78(0.60-1.01)$ & 0.06 & $0.77(0.61-0.96)$ & $<0.05$ & $0.76(0.59-0.98)$ & $<0.05$ & $1.02(0.73-1.42)$ & 0.91 \\
\hline Diuretic exposure (any) & $1.66(1.15-2.41)$ & $<0.01$ & $1.77(1.20-2.61)$ & $<0.01$ & $1.40(0.93-2.10)$ & 0.10 & $1.22(0.84-1.77)$ & 0.30 \\
\hline Furosemide & $1.60(1.12-2.28)$ & $<0.05$ & $1.69(1.14-2.51)$ & $<0.01$ & $1.44(0.99-2.10)$ & 0.05 & $1.19(0.81-1.74)$ & 0.38 \\
\hline Chlorothiazide & $2.15(0.78-5.94)$ & 0.14 & $3.08(1.19-8.00)$ & $<0.05$ & $1.13(0.49-2.61)$ & 0.77 & $2.32(0.95-5.67)$ & 0.06 \\
\hline $\begin{array}{l}\text { CDH repair surgery in } \\
\text { general }\end{array}$ & $1.17(0.90-1.52)$ & 0.23 & $1.13(0.77-1.65)$ & 0.54 & $0.83(0.57-1.19)$ & 0.31 & $1.12(0.90-1.39)$ & 0.29 \\
\hline $\begin{array}{l}\text { CDH repair surgery } \\
\text { on ECLS }\end{array}$ & $1.30(0.81-2.05)$ & 0.28 & $1.20(0.79-1.84)$ & 0.39 & $1.03(0.76-1.39)$ & 0.86 & $0.94(0.69-1.28)$ & 0.71 \\
\hline Abdominal closure surgery & $1.35(1.08-1.70)$ & $<0.01$ & $0.99(0.70-1.40)$ & 0.95 & $0.94(0.69-1.28)$ & 0.68 & $1.05(0.86-1.28)$ & 0.64 \\
\hline Positive blood culture & $2.09(0.87-5.02)$ & 0.10 & $1.05(0.91-1.22)$ & 0.47 & $1.03(0.91-1.18)$ & 0.63 & $0.40(0.04-4.34)$ & 0.45 \\
\hline $\begin{array}{l}\text { Mean arterial pressure } \\
\text { decrease }\end{array}$ & $1.03(1.01-1.06)$ & $<0.05$ & $1.00(0.97-1.02)$ & 0.75 & $1.01(0.98-1.04)$ & 0.70 & $1.00(0.97-1.03)$ & 0.91 \\
\hline
\end{tabular}

$A K I$ acute kidney injury, ECLS extracorporeal life support, $C D H$ congenital diaphragmatic hernia.

${ }^{a}$ Univariable associations between postnatal exposures and AKI, with and without lagged predictors, without adjustment for ECLS use.

${ }^{\mathrm{b}}$ Univariable associations between postnatal exposures and AKI, with and without lagged predictors, adjusted for ECLS.

an increased odds of AKI (Table 3a). Diuretic exposure conferred an increased odds of AKI development, with chlorothiazide exposure at 72,48 , and $24 \mathrm{~h}$ prior to AKI diagnosis and furosemide exposure $24 \mathrm{~h}$ prior to and on the day of AKI diagnosis associated with increased odds of AKI (Table 3a). While CDH repair in general did not confer increased odds of AKI development, the day of abdominal closure was associated with a higher odds of AKI (Table 3a). Three patients had delayed abdominal closure. On the day of AKI diagnosis, each decrease of $1 \mathrm{mmHg}$ in MAP was associated with increased odds of AKI (Table 3a). Notably, the median MAP was $50 \mathrm{mmHg}$ [IQR: $44,54]$ among this cohort. After adjusting for ECLS use, we found that abdominal closure surgery, increased plasma free hemoglobin, diuretic use, and decrements in MAP remained associated with AKI (Table 3b). For those requiring ECLS, an elevated plasma free hemoglobin level $>50 \mathrm{mg} / \mathrm{dL}$ and $>100 \mathrm{mg} / \mathrm{dL}$ was associated with increased odds of AKI on the day of AKI diagnosis, with OR 1.55 (95\% CI:1.02-2.36, $p<0.05)$ and OR 1.24 (95\% CI:1.00-1.53, $p<0.05)$ respectively.

\section{Long-term outcomes}

The overall survival rate of infants with $\mathrm{CDH}$ in this cohort was $79 \%$. Survival was $47 \%$ for those with AKI, while those with no AKI experienced a 98\% survival $(p<0.0001)$ (Fig. 2a). Survival rates decreased with increasing stage of 
a.

\begin{tabular}{|l|l|}
\hline Subgroup & Survival, $n$ (\%) \\
\hline Entire Cohort, $n=90$ & $71(78.8)$ \\
\hline No AKI, $n=56$ & $55(98.2)$ \\
\hline Any AKI, $n=34$ & $16(47.1)^{\star}$ \\
\hline Stage 1 AKI, $n=15$ & $12(80)$ \\
\hline Stage 2 AKI, $n=10$ & $4(40)$ \\
\hline Stage 3 AKI, $n=9$ & $0(0)$ \\
\hline ECLS, $n=32$ & $15(46.8)$ \\
\hline ECLS and no AKI, $n=6$ & $6(100)$ \\
\hline ECLS and AKI, $n=26$ & $9(34.6)$ \\
\hline *(p-value <0.0001 AKI vs No AKI) \\
\hline
\end{tabular}

b.

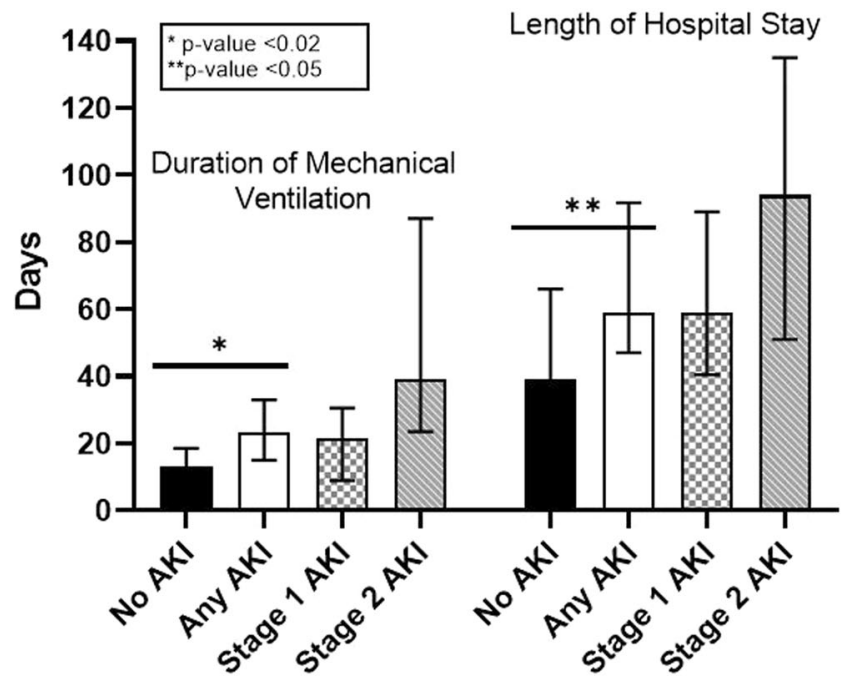

Fig. 2 Effects of AKI on Selected Long-term Outcomes. a Effects of AKI on survival. There was $47 \%$ survival for those with AKI and $98 \%$ survival for those without AKI $(p<0.0001)$. Survival rates decreased with increasing stage of AKI. Of those requiring ECLS, survival was $47 \%$, with $35 \%$ survival for those with AKI vs. $100 \%$ survival for those without AKI. b Effects of AKI on duration of mechanical ventilation and length of hospital stay. Median duration of mechanical ventilation was 13 days [IQR: 8, 18.5] for survivors without $\mathrm{AKI}$ and 23.5 days [IQR: $15.5,33$ ] for survivors with AKI $(p<0.02)$. Median length of hospital stay was 39 days [IQR: 29.5, 66] for survivors without AKI and 59 days [IQR: 47, 91.75] for survivors with AKI $(p<0.05)$. AKI acute kidney injury, ECLS extracorporeal life support.
AKI, with $80 \%, 40 \%$, and $0 \%$ survival with stage 1,2 , and 3 AKI, respectively (Fig. 2a). For those with stage 3 AKI, median age at death was 37 days. Of those requiring ECLS, survival was $47 \%$, with $35 \%$ survival for those with AKI versus $100 \%$ survival for those without AKI (Fig. 2a). For the entire cohort, the unadjusted RR of death for those with AKI compared to those without AKI was $2.1 \quad(95 \%$ CI:1.5-3). For the subset of patients requiring ECLS, the unadjusted RR of death for those with AKI compared to those without AKI was 2.7 (95\% CI:1.4-5.3).

Compared to survivors with AKI, survivors without AKI had a significantly decreased median duration of mechanical ventilation of 13 days [IQR: $8,18.5$ ] versus 23.5 days [IQR: $15.5,33](p<0.02)$ and decreased median length of stay of 39 days [IQR: 29.5,66] versus 59 days [IQR: 47, 91.75] $(p<0.05)$ (Fig. 2b). When stratified by stage of AKI, there was no difference in duration of mechanical ventilation and length of stay (Fig. 2b). CDH survivors who required ECLS demonstrated no significant difference in duration of mechanical ventilation and length of stay for those with and without AKI.

\section{Discussion}

In this single center retrospective study of infants with $\mathrm{CDH}$, AKI was common, occurring in more than a third of patients. Antenatal measures of $\mathrm{CDH}$ severity were significantly worse in those who developed AKI. Postnatally, use of ECLS, exposure to diuretics, abdominal closure surgery, a decrement in MAP, and elevated plasma free hemoglobin were associated with AKI development. Survival among those with AKI was $47 \%$, with increasing stage of AKI associated with decreased survival. AKI was associated with longer duration of hospital stay and mechanical ventilation. This study identified antenatal and postnatal risk factors for AKI in infants with $\mathrm{CDH}$ which warrant further attention at the bedside.

In infants with $\mathrm{CDH}$, the degree of pulmonary hypoplasia by ultrasound or fetal MRI and the presence of liver in the thorax correlate with need for ECLS and mortality [6-11]. In this study, we examined the relationship between antenatal measurements of $\mathrm{CDH}$ severity and $\mathrm{AKI}$, and we found that lower PPLV (<17.5\%), TLV (<25 ml), LHR $(<1)$, O:E LHR $(<38.5 \%)$, and presence of liver in the chest were associated with AKI. While we recognize this is a single-center study, which limits generalizability, providing cutoffs for each of these parameters could help the medical team to risk-stratify patients prior to delivery and early in the hospital course, and AKI preventative strategies can be implemented.

The incidence of AKI in our study (38\%) was comparable to that reported by Ryan et al. (37\%) in a similar cohort, but it was higher than the AWAKEN study (30\%), which represented a more general neonatal population [14, 15]. Within our cohort, infants requiring ECLS had an AKI incidence of $81 \%$, which is higher than that reported by Gadepalli et al. (71\%) in a similar cohort of CDH ECLS infants [4] and higher than the general ECLS neonatal 
population $(63-66 \%)$ [23, 24]. Contrasting our study, Gadepalli et al. utilized the Risk, Injury, Failure, Loss of kidney function, and End-stage kidney disease definition of AKI [4], whereas our study used the Neonatal Modified KDIGO AKI definition (Table 1), a more recently recommended neonatal AKI definition [19, 20]. In addition, SCr can be affected by a positive fluid balance, where creatinine can become diluted, or negative fluid balance, where creatinine can become relatively concentrated [21]. As such, we corrected $\mathrm{SCr}$ for fluid balance [21], potentially more accurately describing the true prevalence of AKI. In our study, ECLS utilization was the greatest risk factor for the development of AKI, suggesting that infants with $\mathrm{CDH}$ requiring ECLS should undergo increased AKI surveillance and renal protective strategies.

Despite efforts to improve CDH outcomes, overall survival remains about $70 \%$ [3]. Comparing survival in infants with and without AKI, we found that AKI was associated with reduced survival (47\% versus 98\%, $p<0.001$ ). In addition, survival rates decreased with increasing stage of AKI. Eight out of nine patients with stage 3 AKI required CRRT. Despite 6 of these infants separating from CRRT and ECLS, no patients with stage 3 AKI survived. The high mortality could have been related to severity of $\mathrm{CDH}$ and ECLS exposure. Using the Extracorporeal Life Support Organization registry, after controlling for multiple variables, AKI and CRRT are independent predictors of mortality, with an adjusted OR 3.2 for death in neonates with AKI and adjusted OR 1.9 for death in neonates requiring CRRT [25]. In our study, ECLS was the greatest predictor for the development of AKI, with an unadjusted RR of death of 2.7 for those with AKI compared to those without AKI. Perhaps targeted AKI prevention strategies and increased AKI surveillance could improve mortality rates, especially in those requiring ECLS.

Utilizing the Extracorporeal Life Support Organization registry, Fleming et al. found that AKI was present at ECLS initiation in 51-64\% of patients and was present by $48 \mathrm{~h}$ in 86-93\% of patients. They concluded that AKI risk factors were likely present prior to ECLS initiation, however they were unable to specifically identify such risk factors [23]. We utilized a lagged predictor model to examine AKI associations, knowing that changes in $\mathrm{SCr}$ concentration can be delayed between the exposure and time to AKI diagnosis [22]. There was an increased odds of AKI with ECLS exposure 72 and $24 \mathrm{~h}$ prior to diagnosis of AKI and on the day of AKI diagnosis. After controlling for ECLS, we demonstrated that diuretic use, abdominal closure surgery, decrements in MAP, and increased plasma free hemoglobin were associated with increased odds of AKI. We believe that these results add meaningful clinical value, as patients with $\mathrm{CDH}$ requiring ECLS represent a high-risk population for morbidity and mortality.
In the AWAKEN cohort, the need for surgical intervention of any kind was associated with higher risk of AKI within and beyond the first postnatal week [26, 27]. The $\mathrm{CDH}$ population, especially those requiring ECLS, represents a surgical challenge due to a potential mismatch between the volume of organs that need to be reduced and the size of the abdominal cavity. This problem is exacerbated by the fluid overload often experienced by patients on ECLS, putting patients at risk for abdominal compartment syndrome after abdominal wall closure. Morozov et al. studied newborns with gastroschisis and $\mathrm{CDH}$, and they found a significant increase in intra-abdominal pressure after abdominal closure with findings consistent with abdominal compartment syndrome [28]. The kidney is vulnerable to increased intra-abdominal pressure, resulting in AKI, due to its position deep within the posterior retroperitoneal space and changes in renal perfusion pressure with decreased arterial blood flow to the kidney and decreased venous blood flow out of the kidney [29]. Even after adjusting for ECLS use, our study found increased odds of AKI with abdominal closure surgery, suggesting consideration of silo placement in patients at risk for abdominal compartment syndrome. Following abdominal closure, we recommend close monitoring of intraabdominal pressure, utilizing bladder pressure as a surrogate, to mitigate the risk of AKI secondary to abdominal compartment syndrome [30]. In the perioperative period, we found that each decrease of $1 \mathrm{mmHg}$ in MAP was associated with increased odds of AKI on the day of AKI diagnosis. Interestingly, despite being decreased from baseline, MAP was still within normal range as defined by gestational age. Therefore, this association with AKI could be related to higher central venous pressure secondary to increased intra-abdominal pressure, leading to decreased renal perfusion pressure.

Furosemide exposure was associated with AKI $24 \mathrm{~h}$ prior to and on the day of AKI diagnosis, whereas chlorothiazide exposure was associated with AKI 72, 48, and $24 \mathrm{~h}$ prior to the diagnosis of AKI. At our institution, in the presence of decreasing UOP, a furosemide challenge is performed $[31,32]$. This is only completed when intravascular fluid status is deemed appropriate. Prior to performing the furosemide challenge, ultrafiltrate is held, central venous pressure and venous inlet pressure trends are utilized to establish fluid status, and furosemide challenge is only performed when there is consensus agreement that the patient is intravascular fluid replete. If there is an inadequate UOP response, chlorothiazide is commonly added as adjuvant therapy. As changes in UOP usually precede the rise in $\mathrm{SCr}$, furosemide challenge is utilized to determine the degree of renal reserve, such that inadequate UOP after furosemide challenge in an intravascularly replete patient is predictive of evolving AKI. In this context, the association 
between diuretic use and AKI found in our analysis could represent tubular dysfunction with later progression to AKI.

Multiple studies have investigated the association between hemolysis, represented by increased plasma free hemoglobin, and AKI [16-18]. In the context of ECLS, hemolysis is caused by exposure of blood to a nonendothelialized circuit and mechanical shear stress within the circuit resulting in release of hemoglobin. Plasma free hemoglobin induces oxidant generation and microcirculatory dysfunction leading to organ hypoperfusion and ischemic damage [16-18]. In a cohort of pediatric patients, Mamikonian et al. found five times greater odds of renal injury with plasma free hemoglobin levels $\geq 100 \mathrm{mg} / \mathrm{dL}$ [17]. In our study, a plasma free hemoglobin level $>50 \mathrm{mg} /$ $\mathrm{dL}$ and $>100 \mathrm{mg} / \mathrm{dL}$ on the day of AKI was associated with an increased odds of AKI.

Limitations to this study include its retrospective nature, allowing us to only establish associations, as well as being a single-center study, which limits generalizability. We did not utilize UOP criteria for AKI diagnosis in the Neonatal Modified KDIGO AKI definition (Table 1), and there might have been episodes of AKI that did not meet $\mathrm{SCr}$ criteria but would have met UOP criteria. However, utilizing UOP to diagnose AKI in neonates has its challenges in that neonates have impaired urinary concentrating abilities, and it is difficult to accurately measure UOP without an indwelling bladder catheter [19]. The patients in our study did not receive daily $\mathrm{SCr}$ measurements, and since the diagnosis of AKI in this study was contingent upon a rise in $\mathrm{SCr}$, delays in diagnosis may have occurred. Additionally, there are inherent challenges with utilizing $\mathrm{SCr}$ to diagnose $\mathrm{AKI}$ in neonates, especially preterm neonates. It is known that an infant's $\mathrm{SCr}$ is largely reflective of maternal $\mathrm{SCr}$ in the first few days of life, and there is an expected gradual decrease in $\mathrm{SCr}$ until a nadir. There is also variability in $\mathrm{SCr}$ related to gestational age, muscle mass, and nutrition [19]. There is ongoing research of urine biomarkers that could potentially diagnose AKI more reliably in this population. Furthermore, cumulative doses of antibiotics and diuretics were not calculated, and therefore the association between cumulative exposures and AKI could not be established. Finally, while there were 90 patients in our study, which is larger than prior similar studies, we were still limited in our statistical analysis by this small sample size and were only able to perform univariable analyses. A larger cohort of patients would allow us to perform multivariate analysis to better understand if certain associations exist.

In conclusion, AKI occurs in more than a third of neonates with $\mathrm{CDH}$, with an incremental increase in mortality with higher stage of AKI. There are limited therapies for treating established AKI, making primary prevention of AKI essential to improving outcomes. This report identifies antenatal and postnatal AKI risk factors and allows for implementation of standardized care bundles geared towards increased kidney function monitoring, limitation of nephrotoxin exposure, and implementation of renal protective strategies which may ultimately reduce AKI incidence and severity.

Acknowledgements We acknowledge John Kinsella, MD and Ken Liechty, MD for their knowledge, expertise, and contribution to the care of these complex patients as part of the $\mathrm{CDH}$ team at Children's Hospital Colorado.

Author contributions KMG, DES, MJK, and JG conceptualized and designed the study. BML, MJK, and JG acquired the data, and BML, JTB, and JG analyzed the data. BML, JTB, KMG, and JG interpreted the data. BML, JTB, and JG drafted the paper. All authors revised the paper. All authors approved the final version of the manuscript and agreed to be accountable for all aspects of the work.

\section{Compliance with ethical standards}

Conflict of interest The authors declare no competing interests.

Publisher's note Springer Nature remains neutral with regard to jurisdictional claims in published maps and institutional affiliations.

\section{References}

1. Benjamin JR, Bizzarro MJ, Cotton CM. Congenital diaphragmatic hernia: updates and outcomes. NeoReviews. 2011;12:e439-452.

2. Lagnham MR,Jr, Kays DW, Ledbetter DJ, Frentzen B, Sanford LL, Richards DS. Congenital diaphragmatic hernia. Epidemiology and outcome. Clin Perinatol. 1996;23:671-88.

3. Grover TR, Rintoul NE, Hedrick HL. Extracorporeal membrane oxygenation in infants with congenital diaphragmatic hernia. Semin Perinatol. 2018;42:96-103.

4. Gadepalli SK, Selewski DT, Drongowski RA, Mychaliska GB. Acute kidney injury in congenital diaphragmatic hernia requiring extracorporeal life support: an insidious problem. J Pediatr Surg. 2011;46:630-35.

5. Murthy K, Pallotto EK, Gien J, Brozanski BS, Porta NFM, Zaniletti I, et al. Predicting death or extended length of stay in infants with congenital diaphragmatic hernia. J Perinatol. 2016;36:654-59.

6. Benachi A, Cordier AG, Cannie M, Jani J. Advances in prenatal diagnosis of congenital diaphragmatic hernia. Semin Fetal Neonatal Med. 2014;19:331-37.

7. Jani JC, Nicolaides KH, Gratacós E, Vandecruys H, Deprest JA, FETO Task Group. Fetal lung-to-head ratio in the prediction of survival in severe left-sided diaphragmatic hernia treated by fetal endoscopic tracheal occlusion (FETO). Am J Obstet Gynecol. 2006;195:1646-50.

8. Barnewolt CE, Kunisaki SM, Fauza DO, Nemes LP, Estroff JA, Jennings RW. Percent predicted lung volumes as measured on fetal magnetic resonance imaging: a useful biometric parameter for risk stratification in congenital diaphragmatic hernia. J Pediatr Surg. 2007;42:193-97.

9. Lee TC, Lim FY, Keswani SG, Frischer JS, Haberman B, Kingma PS, et al. Late gestation fetal magnetic resonance imagingderived total lung volume predicts postnatal survival and need for extracorporeal membrane oxygenation support in isolated congenital diaphragmatic hernia. J Pediatr Surg. 2011;46:1165-71. 
10. Deprest JA, Flemmer AW, Gratacos E, Nicolaides K. Antenatal prediction of lung volume and in-utero treatment by fetal endoscopic tracheal occlusion in severe isolated congenital diaphragmatic hernia. Semin Fetal Neonatal Med. 2009;14:8-13.

11. Jani J, Nicolaides KH, Keller RL, Benachi A, Peralta CFA, Favre $\mathrm{R}$, Antenatal-CDH Registry Group. et al. Observed to expected lung area to head circumference ratio in the prediction of survival in fetuses with isolated diaphragmatic hernia. Ultrasound Obstet Gynecol. 2007;30:67-71.

12. Hoste EA, Bagshaw SM, Bellomo R, Cely CM, Colman R, Cruz $\mathrm{DN}$, et al. Epidemiology of acute kidney injury in critically ill patients: a multinational AKI-EPI study. Intensive Care Med. 2015;41:1411-23.

13. Kaddourah A, Basu RK, Bagshaw SM, Goldstein SL, AWARE Investigators. Epidemiology of acute kidney injury in critically ill children and young adults. N Engl J Med. 2017;376:11-20.

14. Jetton JG, Boohaker LJ, Sethi SK, Wazir S, Rohatgi S, Soranno DE, et al. Incidence and outcomes of neonatal acute kidney injury (AWAKEN): a multicenter, multinational, observational cohort study. Lancet Child Adolesc Health. 2017;1:184-94.

15. Ryan A, Gilhooley M, Patel N, Reynolds BC. Prevalence of acute kidney injury in neonates with congenital diaphragmatic hernia. Neonatology. 2020;117:88-94.

16. Kim-Campbell N, Gretchen C, Callaway C, Felmet K, Kochanek $\mathrm{PM}$, Maul T et al. Cell-free plasma hemoglobin and male gender are risk factors for acute kidney injury in low risk children undergoing cardiopulmonary bypass. Crit Care Med. 2017. https://doi.org/10.1097/CCM.0000000000002703.

17. Mamikonian LS, Mamo LB, Smith PB, Koo J, Lodge AJ, Turi JL et al. Cardiopulmonary bypass is associated with hemolysis and acute kidney injury in neonates, infants, and children. Pediatr Crit Care Med. 2014. https://doi.org/10.1097/PCC.0000000000000047.

18. Vermeulen Windsant IC, Hanssenn SJ, Buurman WA, Jacobs MJ. Cardiovascular surgery and organ damage: time to reconsider the role of hemolysis. J Thorac Cardiovasc Surg. 2011;142:1-11.

19. Zappitelli M, Ambalavanan N, Askenazi DJ, Moxey-Mims MM, Kimmel PL, Star RA, et al. Developing a neonatal acute kidney injury research definition: a report from the NIDDK neonatal AKI workshop. Pediatr Res. 2017;82:569-73.

20. Harer MW, Charlton JR, Tipple TE, Reidy KJ. Preterm birth and neonatal acute kidney injury: implications on adolescent and adult outcomes. J Perinatol. 2020;40:1286-95.

21. Liu KD, Thompson BT, Ancukiewicz M, Steingrub JS, Douglas IS, Matthay MA, et al. National Institutes of Health National Heart, Lung, and Blood Institute Acute Respiratory Distress Syndrome Network: Acute kidney injury in patients with acute lung injury: impact of fluid accumulation on classification of acute kidney injury and associated outcomes. Crit Care Med. 2011;39:2665-71.

22. Askenazi DJ, Koralkar R, Levitan EB, Goldstein SL, Devarajan P, Khandrika $S$, et al. Baseline values of candidate urine acute kidney injury biomarkers vary by gestational age in premature infants. Pediatr Res. 2011;70:302-6.

23. Fleming GM, Sahay R, Zappitelli M, King E, Askenazi DJ, Bridges $\mathrm{BC}$, et al. The incidence of acute kidney injury and its effect on neonatal and pediatric extracorporeal membrane oxygenation outcomes: a multicenter report from the kidney intervention during extracorporeal membrane oxygenation study group. Pediatr Crit Care Med. 2016;17:1157-69.

24. Zwiers AJM, de Wildt SN, Hop WCJ, Dorresteijn EM, Gischler SJ, Tibboel D, et al. Acute kidney injury is a frequent complication in critically ill neonates receiving extracorporeal membrane oxygenation: a 14-year cohort study. Crit Care. 2013;17: R151.

25. Askenazi DJ, Ambalavanan N, Hamilton K, Cutter G, Laney D, Kaslow $\mathrm{R}$ et al. Acute kidney injury and renal replacement therapy independently predict mortality in neonatal and pediatric noncardiac patients on extracorporeal membrane oxygenation. Pediatr Crit Care Med. 2011. https://doi.org/10.1097/PCC. 0b013e3181d8e348.

26. Charlton JR, Boohaker L, Askenazi D, Brophy PD, Fuloria M, Gien J, et al. Late onset neonatal acute kidney injury: results from the AWAKEN Study. Pediatr Res. 2019;85:339-48.

27. Charlton JR, Boohaker L, Askenazi D, Brophy PD, D'Angio C, Fuloria $\mathrm{M}$, et al. Incidence and risk factors of early onset neonatal AKI. Clin J Am Soc Nephrol. 2019;14:184-95.

28. Morozov D, Morozova O, Pervouchine D, Severgina L, Tsyplakov A, Zakharova N, et al. Hypoxic renal injury in newborns with abdominal compartment syndrome (clinical and experimental study). Pediatr Res. 2018;83:520-26.

29. De Waele JJ, De Laet I, Kirkpatrick AW, Hoste E. Intraabdominal hypertension and abdominal compartment syndrome. Am J Kidney Dis. 2011;57:159-69.

30. Laje P, Hedrick HL, Flake AW, Adzick NS, Peranteau WH. Delayed abdominal closure after congenital diaphragmatic hernia repair. J Pediatr Surg. 2016;51:240-43.

31. Sharma A, Mucino MJ, Ronco C. Renal functional reserve and renal recovery after acute kidney injury. Nephron Clin Pract. 2014;127:94-100.

32. Chawla LS, Ronco C. Renal stress testing in the assessment of kidney disease. KI Rep. 2016;1:57-63. 